International Journal of Advanced Trends in Computer Science and Engineering

Available Online at http://www.warse.org/IJATCSE/static/pdf/file/ijatcse4091.32020.pdf

https://doi.org/10.30534/ijatcse/2020/4091.32020

\title{
Cloud based Petrol Pump Blacklisting using IoT
}

\author{
Rahul Jimmy ${ }^{1}$, Shone Thomas $^{2}$, Suraj P. Nair ${ }^{3}$, Vishnu Baiju ${ }^{4}$, Uday Babu P ${ }^{5}$ \\ ${ }^{1}$ Department of Computer Science, Rajagiri School of Engineering and Technology, Kerala, India, \\ rahuljimmy6@gmail.com \\ ${ }^{2}$ Department of Computer Science, Rajagiri School of Engineering and Technology, Kerala, India, \\ shonethomas111999@gmail.com \\ ${ }^{3}$ Department of Computer Science, Rajagiri School of Engineering and Technology, Kerala, India, \\ surajpnair98@gmail.com \\ ${ }^{4}$ Department of Computer Science, Rajagiri School of Engineering and Technology, Kerala, India, \\ vishnubaiju083@gmail.com \\ ${ }^{5}$ Department of Computer Science, Rajagiri School of Engineering and Technology, Kerala, India, \\ udaybabup@gmail.com
}

\begin{abstract}
According to various statistics and reports, the number of frauds occurring in petrol pumps is very high and the general public is ignorant. To solve these issues, we propose a novel cloud based storage scheme in combination with IoT sensors, whose front end is an Android application. The customer and the dealer enter the data about the fuel being filled into the tank through the application. A sensor placed in the petrol tank measures the petrol level before and after pumping, and the difference in levels is calculated. The data comprising of petrol price, amount to be fuelled for, petrol pump id, and a unique transaction id is uploaded to the cloud. The data pushed into the cloud is checked for detecting fraudulent practices in petrol pumps. Detection of any malpractices reduces the trust factor of the pump leading, and even could lead to its blacklisting which is then displayed in the map. The trust factor of every petrol pump in the country will be marked on a map which is accessible through the Android application.
\end{abstract}

Key words: Blockchain, Cloud Storage, Internet of Things (IoT), Web-scraping

\section{INTRODUCTION}

Fuel is one of the most widely used sources of energy in the world today. The energy produced by burning fuel has many applications, such as powering vehicles, ships, and airplanes as well as providing electricity for homes and buildings. Some common types of fuels are petro fuel, gas oil, diesel fuel, fuel oils, aviation fuel, jet fuel, and marine fuels.

Because of its many uses, fuel is a critical component of the global economy. Due to the incredibly high demand for fuel, the number of malpractices done from the initial stage of extraction, till it reaches the end customers are very high.

To reduce the number of these frauds by empowering the public is our key objective. This paper proposes a system consisting of an Android application as front end which makes use of with the support of cloud storage and the IoT for efficient implementation.

The Internet of things is a system of interrelated computing devices, mechanical and digital machines provided with unique identifiers and the ability to transfer data over a network without requiring human-to-human or human-to-computer interaction.

\section{LITERATURE REVIEW}

There have been a number of IoT systems created and implemented for various purposes, with different system architectures. The IoT system proposed in [1] is a Smart Petrol pump. This system maintains fuel levels, if and when fuel in a petrol station falls below a particular level, the central will provide fuel to that corresponding station. Another aspect is website which is used by both admin and users, to maintain information regarding the fuel levels at various fuel stations. The sensor used is ultrasonic. The proposed system in [2] and [3] makes use of flow meters to detect the amount of fuel entering and uses a cloud to store this information. A detailed analysis of fuel consumption is made and results are made available to the user in [2] and using this data frauds are detected. [6] and [2] was also created with the aim of reducing the thefts and illicit practices occurring in the petrol pumps.

IoT system in [4] was created with aim of detecting anomalies in oil and gas industries. Using IoT we are able to detect leaks in pipes and this information is then made known to the maintenance staff. Real time monitoring of these pipes increases efficiency in problem detection. Using these 
Rahul Jimmy et al., International Journal of Advanced Trends in Computer Science and Engineering, 9(1.3), 2020, 267 - 273

systems, faults are dealt with much faster thereby saving both time and money. The proposed system in [5] makes use of float sensor. This system also consists of an Arduino UNO board, mileage calculation is done and is displayed on a LCD display. These projects and papers have provided us with inspiration to come up with an integrated version, and are improved upon using upcoming technology such as cloud storage.

\section{OBJECTIVES}

\section{Primary Objective}

To blacklist those petrol pumps which uses fraudulent methods thereby reducing corruption using a real-time fuel monitoring IoT system, with the support of a cloud storage and android applications as its front end.

\section{Secondary Objectives}

- To help the public from falling victim to the fraudulent methods they face which are unknown to them while refilling their vehicles in gas stations.

- Preventing petrol pumps from tax evasion.

- Create a trustworthy rating system for petrol pumps.

- Mark blacklisted petrol pumps in Google Maps.

- Enable users to identify nearby trustworthy petrol pumps.

- To avoid the customer's overload on looking at the fuel refill display all the time of refilling.

\section{PROPOSED SYSTEM \& ARCHITECTURE}

The system is a combination of both hardware and software. Each part is integral as shown below.

\section{Problem definition}

The root of the problem lies in the inefficiency of Ministry of Petroleum and the lack of resources to conduct routine checks on the operations of petrol pumps across the nation. This is exploited by dealers present in the petrol pumps to cheat the customers by various methods. Our aim is to negate this issue by empowering the customers. The idea is to use an application to validate transactions by input from dealer, customer and IoT devices placed in the fuel tank. The data collected is then stored in a cloud where the data is used to assess the trustworthiness of the petrol pumps. This information is then used to blacklist those petrol pumps which are fraudulent in nature, and made known to the general public, thereby increasing the sales of petrol pumps which do not indulge in malpractices.

\subsection{Methodology}

The method can be primarily divided into three sections namely, Android app, Proceedings at the pump and the IoT framework in the tank.

\subsubsection{Android Applications}

For better performance and for the ease of development both dealer and customer are provided with separate applications. There are certain aspects which are common to both the applications including databases which store information regarding the users of the respective applications. This database is stored online in Firebase. Any maintenance in the application is done by the admin.

\section{a) Dealer Application:}

While registering the dealer (petrol pump), enters the location of the petrol pump. The cost of the fuel is based on where it is located and changes daily. Therefore the first purpose of this application is to obtain the latest rates using web-scraping. The dealer then enters, the amount to be fuelled for, as requested by the customer. The application then generates a $Q R$ code with the following Petrol-pump id, transaction id, petrol price and amount to be fuelled for.

b) Customer Application:

The customer logs into his account, and consists of a $\mathrm{QR}$ code scanner. He then scans the $\mathrm{QR}$ code generated by the dealer, and checks if the amount entered by the dealer is as per his requirements. Once this is made sure and fuelling is done, the information obtained from the dealer and data from the sensors are pushed into the cloud (Firebase) where the data is cross checked. If any disparity is observed, the trustworthiness of the corresponding petrol pumps is updated. These changes are finally updated and made known to the customers using Google Map API.

\subsubsection{Proceedings at the pump}

(i) The customer requests the dealer to refill his vehicle for a certain amount of fuel.

(ii) The dealer enters amount into his app. QR code is generated

(iii) Customer scans QR code, obtains transaction details and verifies if amount entered is correct.

(iv) Once verified, the dealer will re-fuel the customer's vehicle upto requested amount of fuel.

(v) The sensor fitted in the vehicle's petrol tank will detect the change in fuel level, which is the difference between the initial level (before fuelling) and final level (after fuelling). Then this data is used to calculate the amount for which the vehicle has been re-fuelled. This information is then pushed onto the cloud.

(vi) The customer pays the bill amount and this marks the end of a transaction

\subsubsection{IoT Framework}

The IoT framework is very basic and easy to implement consisting of a Bluetooth module and ultrasonic sensor. The changes in the levels obtained by the sensor are sent to the customer application using a Bluetooth module. 


\subsection{Hardware}

\section{a) Arduino}

The sensor acquisition in this work is done using Arduino. The Arduino version considered is Arduino UNO (Figure 1). Arduino is an open-source hardware and software company, user community and project that designs and manufactures single-board micro-controllers and kits for building digital devices.

Arduino board designs use a variety of microprocessors and controllers. The boards are equipped with sets of digital and analog input/output $(\mathrm{I} / \mathrm{O})$ pins. The micro-controllers can be programmed using $\mathrm{C}$ and $\mathrm{C}++$ programming languages.

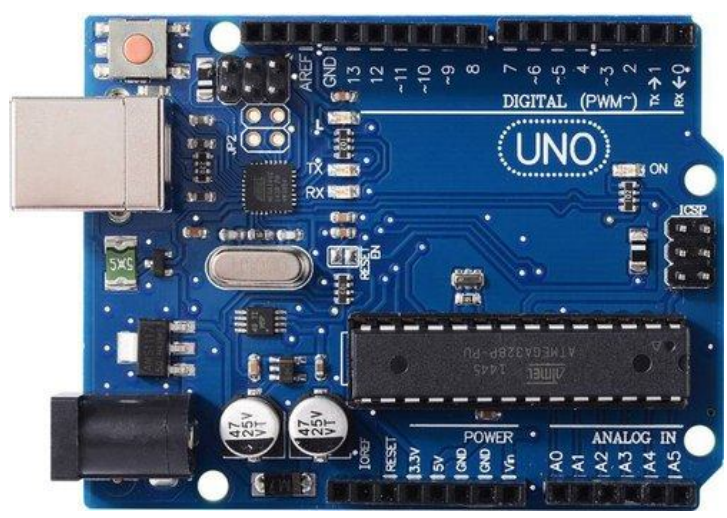

Figure 1: Arduino UNO

It has 6 analog input pins and 14 digital input/output pins. The operating voltage is $5 \mathrm{~V}$ and input voltage range is 7 $12 \mathrm{~V}$. Arduino IDE is required for programming. Arduino UNO should be connected to computer with USB cable to be programmed through USB-to-serial converter.

\section{b) Ultrasonic Sensor}

As the name indicates, ultrasonic / level sensors measure distance by using ultrasonic waves. The sensor head emits an ultrasonic wave and receives the wave reflected back from the target (figure 2). Ultrasonic / level sensors measure the distance to the target by measuring the time between the emission and reception. An ultrasonic / level sensor uses a single ultrasonic element for both emission and reception.

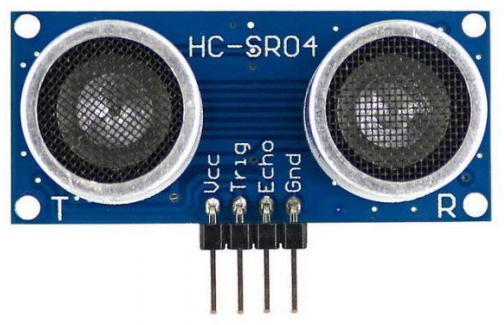

Figure 2: Ultrasonic Sensor
Distance Calculation (Figure 3) : Distance $\mathrm{L}=1 / 2 \times \mathrm{T} \times \mathrm{C}$, where $\mathrm{L}$ is the distance, $\mathrm{T}$ is the time between the emission and reception, and $\mathrm{C}$ is the sonic speed. (The value is multiplied by $1 / 2$ because $\mathrm{T}$ is the time for go-and-return distance.)

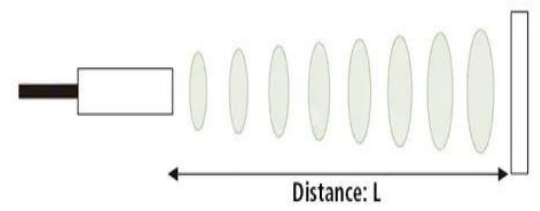

Figure 3: Distance Calculation

\section{c) Bluetooth Module: (HC -04)}

The Bluetooth module (figure 4) has 4 connections, GND, $5 \mathrm{~V}, \mathrm{RX}$ and TX. The TX pin on the Bluetooth module connects to the RX pin on the Arduino and vice versa. For serial communication, a transmit (TX) connection need to be received by a $(\mathrm{RX})$ connection.

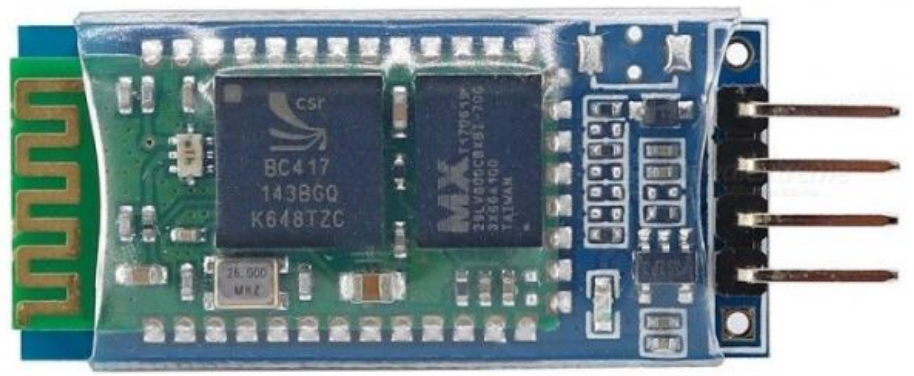

Figure 4: Bluetooth module

\subsection{Software}

a) Firebase

Firebase is the cloud platform that we use for this work. It provides a real-time database and backend as a service. The service provides application developers an API that allows application data to be synchronized across clients and stored on Firebase's cloud.

\section{b) Android Studio:}

Android Studio is the official integrated development environment (IDE) for Google's Android operating system, built on JetBrains IntelliJ IDEA software and designed specifically for Android development. The application we have written is in Java.

\subsection{System Architecture}

IOT based fuel monitoring and tracking system has implemented for overcome Fraud at petrol-pumps. The fuel tank of the vehicles is fitted with ultrasonic sensors that measure the depth. The sensor measures the initial depth and the depth after the fuel is filled. The data is then passed on to 
Arduino board: The dimensions of the fuel tank are initially programmed into the board and the depth change is used to find the volume change. The data is then pushed into the application using Bluetooth module. This data once verified is then pushed onto the cloud.

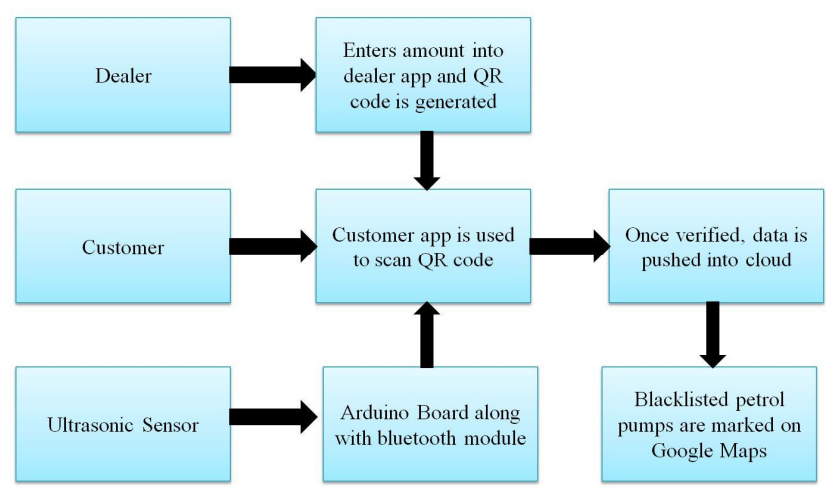

Figure 5: System Overview

The IoT framework mainly consists of the ultrasonic sensor (figure 6) (HC-SR04), Bluetooth module (HC-06) and Arduino board UNO r3.

The petrol pump operator provides a bill to the customer that contains the details of the current transaction. Each petrol station is provided with a unique id. The operator inputs the requested amount by the customer into the mobile application and QR code is generated. The customer scans this QR code, verifies the data and this is pushed onto the cloud. Each block contains details of a particular transaction and points to another block which contains details of another transaction. The data from the vehicle fuel tank sensor is then multiplied with the fuel price of one liter which is obtained by web scrapping and is cross checked with data from customer's and pump operator's mobile application and the trustworthy of particular pump is evaluated.

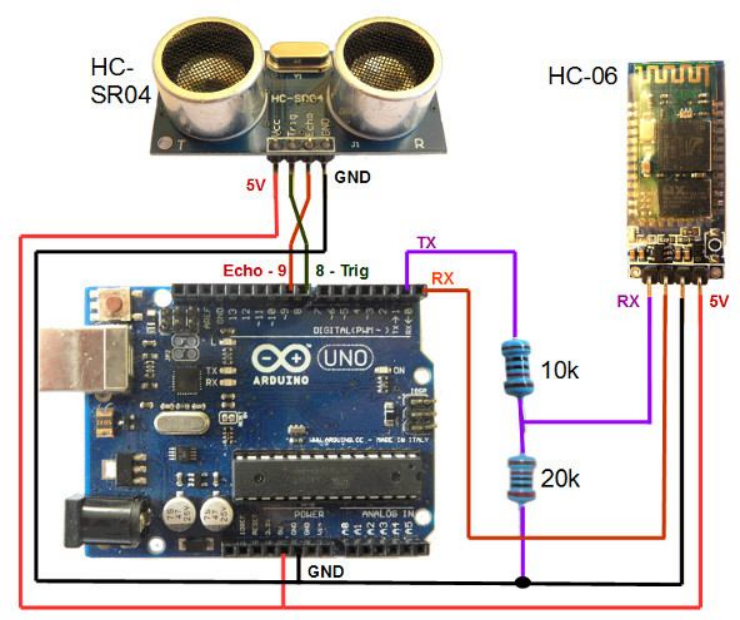

Figure 6: Ultrasonic Sensor Circuit
The trustworthy of the pump for each transaction calculated by:

Trustworthy $\%=(($ value from fuel tank sensor $*$ current fuel liter price) / (amount entered by the customer)) * 100

In the cloud, a database is also maintained. The database includes details about pump id, number of transactions, current average trustworthy percentage of the pump and a yes or no column stating whether the pump is blacklisted or not. The average trustworthy percentage is calculated by:

Avg trustworthy $\%=(($ current trustworthy $\% *$ no of transaction $)+($ new trustworthy $\%)) /($ new total number of transactions)

The database is then updated with the new values and the current trustworthy \% is then compared with the values in the trustworthy table and is then blacklisted if rating falls below threshold. Figure $7-13$ shows the various screenshots of the system.

\section{RESULTS AND IMPLEMENTATION}

\section{Algorithm for Android application for Customer Step1: Start \\ Step2: If NOT REGISTERED YET, go to Registration Page}

Step2.1: Enter Name

Step2.2: Enter Password

Step2.3: Enter E-mail id

Step2.4: Confirm E-mail id and REGISTER

Step3: If ALREADY REGISTERED, go to Login page

Step3.1: Enter UserName

Step4: After Login Customer enters the amount of fuel he requested.

Step5: He then scans the QR code generated by the dealer using his dealer application to obtain the petrol rate for that locale and application calculates the amount of fuel that will enter the tank.

Step6: Customer verifies the amount entered by the dealer, and this data is then pushed into the cloud.

Step 7: Stop

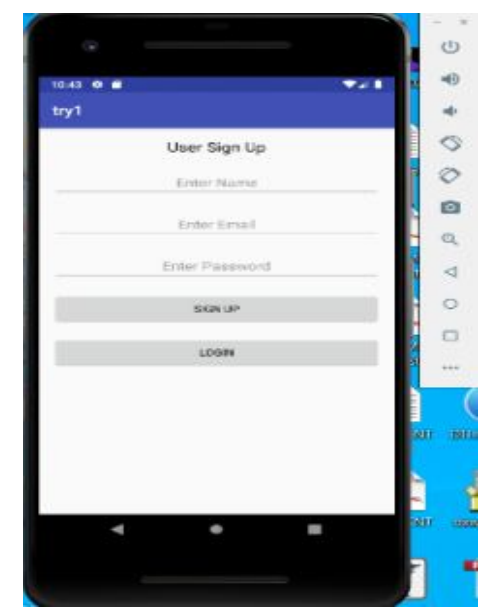

Figure 7: Customer sign up page 
Rahul Jimmy et al., International Journal of Advanced Trends in Computer Science and Engineering, 9(1.3), 2020, 267 - 273

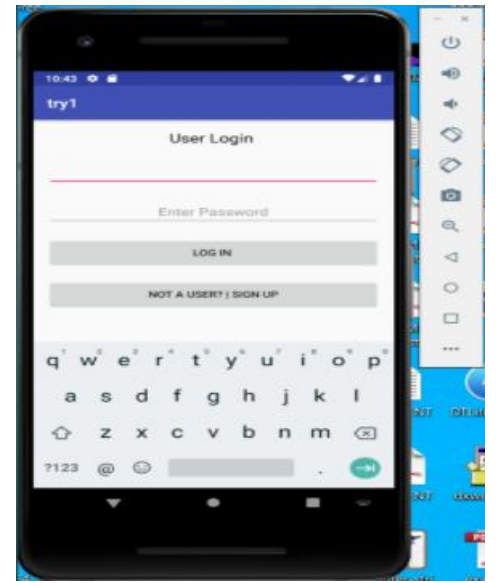

Figure 8: Customer Login page

After logging in, customer enters the amount of fuel he requested.

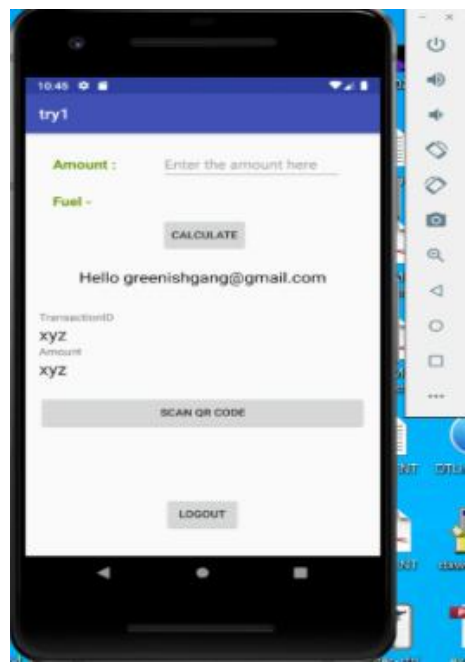

Figure 9: User Page

The data is analyzed and the blacklisted petrol pumps are shown on the map.

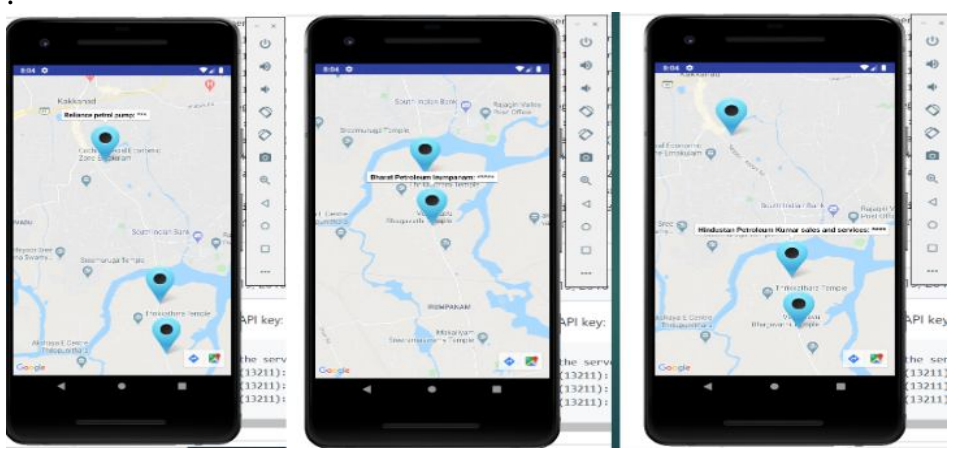

Figure 10: Petrol Pumps marked on Google Maps

\section{Algorithm for Android Application of dealer}

Step1: Start

Step2: If NOT REGISTERED YET, go to Registration Page Step2.1: Enter Name

Step2.2: Enter Password

Step2.3: Enter E-mail id

Step2.4: Enter District

Step 3: If already registered, go to login page, enter mail and password.

Step 4: The application obtains real time rates of the petrol based upon the district the pump is located. For this purpose we make use of web-scraping.

Step 5: QR code is generated with the following Petrol-pump id, transaction id, petrol price and amount to be fuelled for. Step 6: This QR code is then scanned by the customer by means of his app.

Step 7: Stop

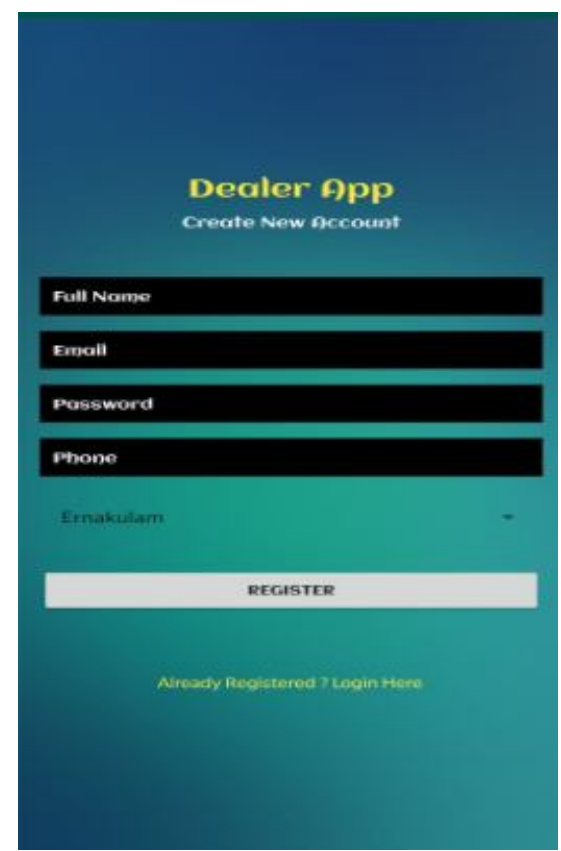

Figure 11: Dealer Login Application 


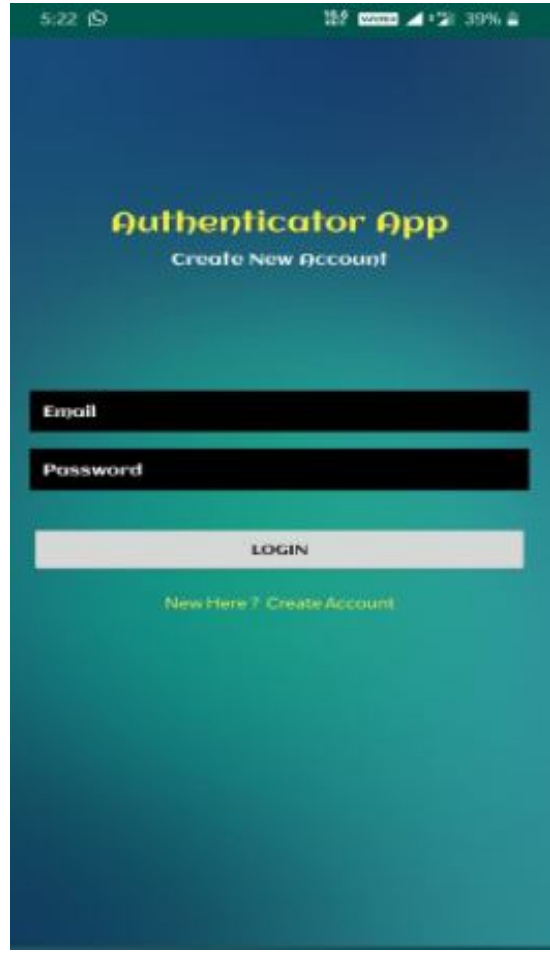

Figure 12: Login page

The latest price of the petrol is obtained from the net using web-scraping, dealer enters the requested amount and application generates the QR code.

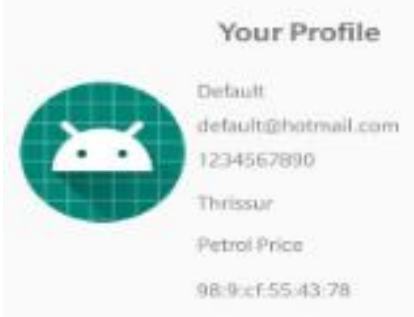

GENERATE QR

LOGOUT

Figure 13: User Page
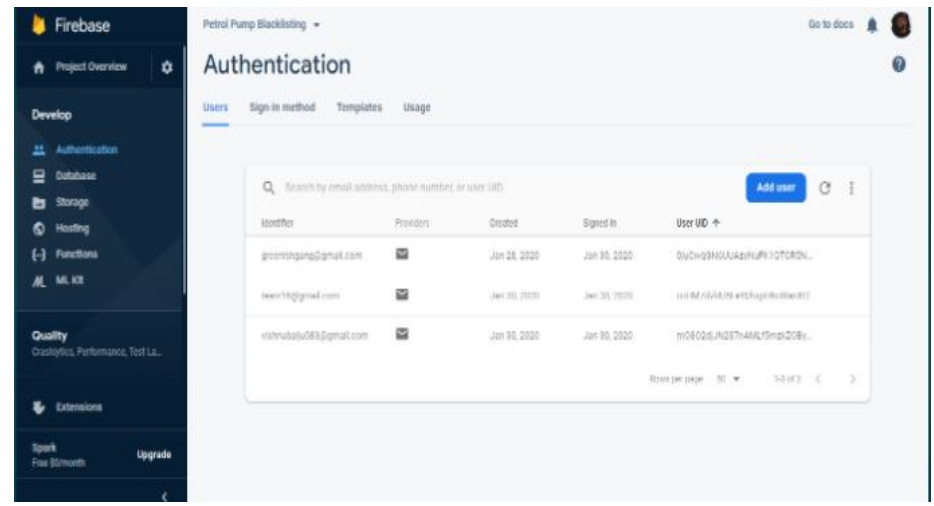

Figure 14: Firebase

Firebase (cloud storage) is used to store the information of the petrol pumps, customers registered and also the details of the transaction.

\section{CONCLUSION}

Fraud and malpractices are very common in almost every industry, including petrol pumps. This project aims to reduce the corruption involved and malpractices done in the petrol pumps, by enabling the customers to verify and rate the services provided at the petrol pumps. After execution of the project, petrol pumps across the state will be assessed based upon the services provided. Through a rating based system, petrol pumps are blacklisted, and this is made known through an android application and later marked on to Google Maps.

\section{FUTURE SCOPE}

There are plenty of opportunities for future work in this field. For instance, fuelling can be automated by installation of chips within petrol pump and fuel tank, removing the middle man (dealer). In this scenario, payment is also automated. Money is directly transferred from customer's account to that of the petrol pump. This is done by linking the chips to their corresponding user's accounts. Another improvement will be adding mechanisms to test for the purity of the fuel being used.

To increase security and integrity a blockchain framework can be used. Smart contract could be written in Solidity and deployed on Ethereum. We can also make use of a self-hosted wallet, Meta mask and Infura that allows us to run Ethereum dApps on our browser without running a full Ethereum node. The transactions can be tracked using a block explorer called Etherscan 


\section{REFERENCES}

[1] Punit Gupta, Sawan Patodiya, Digvijaym Singh, Jasmeet Chhabra, Achman Shukla, IoT based Smart Petrol Pump (2016 Fourth International Conference on Parallel, Distributed and Grid Computing PDGC). https://doi.org/10.1109/PDGC.2016.7913168

[2] Vijayakumar P., Ganesan V., Pratik Patwari, Rajnandini Singh, Sharmila A., Payal P. Tayade, R. Rajashree, M. Tamilselvi, IoT Based Smart Fuel Monitoring System (International Journal of Recent Technology and Engineering (IJRTE) ISSN: 2277-3878, Volume-8 Issue-2, July 2019).

https://doi.org/10.35940/ijrte.A1146.078219

[3] Pavan Manjunath, Pritam Gajkumar, IoT Based Handy Fuel Flow Measurement, (Proceedings of the Third International Conference on I-SMAC (IoT in Social, Mobile, Analytics and Cloud) (I-SMAC 2019) IEEEn Xplore Part Number: CFP19OSV-ART https://doi.org/10.1109/I-SMAC47947.2019.9032467

[4] Wazir Zada Khan, Mohammed Y Aalsalem, Muhammad Khurram Khan, Md. Shohrab Hossain and Mohammed Atiquzzaman, A Reliable Internet of Things based Architecture for Oil and Gas Industry, ICACT2017 February $19 \sim 22,2017$.

[5] Dr.Anuradha, B.Priyadharsini, A.Yuvasri, M.Yamuna, Fuel Level Indication and Mileage Calculator Using IoT, 2019 5th International Conference on Advanced Computing \& Communication Systems (ICACCS). https://doi.org/10.1109/ICACCS.2019.8728360

[6] Oluwatobi Oyinlola, IoT stop fuel theft in Africa, IEEE Newsletter May 2017 